\title{
Variation in Organic Acid, Sugar and Phenolic Compounds in Fruits of Historical Apple Cultivars
}

\section{Ferit CELIK ${ }^{1}$, Muttalip GUNDOGDU ${ }^{2}$, Sezai ERCISLI ${ }^{3 *}$, Baris KAKI ${ }^{4}$, Selma BERK ${ }^{5}$, Gulce ILHAN ${ }^{3}$, Halil Ibrahim SAGBAS ${ }^{3}$}

\author{
${ }^{1}$ Yuzuncu Yil University, Faculty of Agriculture, Department of Horticulture, Van, Turkey; femcelik@hotmail.com \\ ${ }^{2}$ Abant Izzet Baysal University, Faculty of Agriculture and Natural Sciences, Department of Horticulture, Bolu, Turkey; \\ gundogdumuttalip@gmail.com \\ ${ }^{3}$ Ataturk University, Faculty of Agriculture, Department of Horticulture, Erzurum, \\ Turkey; sercisli@gmail.com(*correspondingauthor); halil.sagbas@atauni.edu.tr; gulce.ilhan@atauni.edu.tr \\ ${ }^{4}$ Usak University, Faculty of Economics and Administrative Sciences, Department of Econometrics, Usak, Turkey; baris.kaki@usak.edu.tr \\ ${ }^{5}$ Abant Izzet Baysal University, Mudurnu Sureyya Astarci Vocational School, Department of Plant and Animal Production, Bolu, \\ Turkey; kuru_s@ibu.edu.tr
}

\begin{abstract}
Knowledge about diversity of local apple cultivars is particularly important and helps to ensure that specific cultivars are available when and where farmers need them. The phenolic content, organic acids and sugars were investigated in the four historical apple cultivars ('Aslik', 'Cebegirmez', 'Bey Elmasi' and 'Arapkizi') grown in Van province, located in Eastern Anatolia, Turkey. In the study, ellagic acid $(63.034 \mathrm{mg}$ per $100 \mathrm{ml})$ was found to be higher than other phenolic compounds and followed by vanilic acid, caffeic acid and routine, respectively. When examined the apple cultivars, it has been found that the 'Bey Elmasi' cultivar has the highest content of six phenolic acids (chlorogenic, ferulic, $o$-coumaric, $p$-coumaric, catechin and quercetin). The organic acid contents were as follows: malic $>$ fumaric $>$ succinic $>$ citric $>$ tartaric among cultivars. 'Arapkizi' cultivar possessed the highest value of four organic acids except of tartaric acid. It was determined that fructose was the major sugar for all apple cultivars. There was a positive correlation among gallic, ellagic acid and routine. Similarly, the positive correlation was observed among malic, fumaric and succinic acid. However the negative correlation between citric acid and all sugars was observed. As a result, 'Bey Elmasi' and 'Arapkizi' cultivars with higher phenolic content, 'Arapkizi' cultivar with higher organic acid content and 'Aslik' cultivar with higher sugar content comes to the foreground. Results indicated the importance of local apple germplasm to use of them for future apple breeding activities to increase inner quality.
\end{abstract}

Keywords: autochthonous Turkish apple cultivars; organic acids; phenolic compounds; sugars

\section{Introduction}

Anatolia is accepted the homeland of many wild, wild edible and cultivated fruit species (Kamiloglu et al., 2009; Tosun et al., 2009; Ercisli et al., 2012; Canan et al., 2016; Caliskan et al., 2017). Anatolia has also rich apple genetic resources including mostly historical cultivars selected by humans among wild populations for centuries. There are hundreds historical apple cultivars distributed different agroclimatic conditions in Turkey. This kind of richness gives the choice of different uses of them for the breeders (Kaya and Balta, 2013). In addition Turkey is an important apple producer in the world besides the rich apple genetic resources. World apple production is more than 84 million tons. Turkey produces around 3.0 million tons and takes fourth place after China (40 million), United States (5 million) and Poland (3.1 million) (FAO, 2017).

In recent years their consumption are increasing as a diet product and for health because advertisement of apple phytochemicals (Boyer and Liu, 2004). They reduce risk of lung cancer (Michels et al., 2006), risk of type II diabet and lost weight (Boyer and Liu, 2004) and prevent risk of cardiovascular disease (Sesso et al., 2003; Boyer and Liu, 2004). The apples are rich in potassium, phosphorus, calcium, magnesium and sodium, respectively (Mordogan and Ergun, 2001). Also, apple fruits have strong antioxidant activity due to contained flavonoid and phenolic acids (Wolfe et al., 2003; Boyer and Liu, 2004). The researchers suggest that the apple fruit is rich in chlorogenic, catechin (Carbone et al., 2011) and phloridzin (Jakobek et al., 2016). 
The apple peel is richer in phenolic substance than apple flesh (Wang et al., 2015; Khanizadeh et al., 2008). Apple seeds are also known to be rich in phenolic compounds. It is emphasized by some researchers that the seeds contain the most phloridzin and followed by hyperin and chlorogenic acid (Xu et al., 2016). Studies show that apple growing conditions, storage time and shape (Seçmen et al., 2015) and cultivar affect phenolic compounds (Khanizadeh et al., 2008).

It is important to protect apple genetic resources in order to obtain qualified apple cultivars. The value of the historical cultivars grown in our country must be preserved and the biological characteristics should be determined. Van province is home to many fruit species and the presence of a large number of historical apple cultivars (Özrenk et al., 2011). Although there are some studies on the pomological characteristics of these historical cultivars, no studies on their phytochemical properties have been reported.

Thus, the aim of our study was to reveal the phytochemical properties of 4 well-known historical apple cultivars grown in the Van region. In addition, the correlation between phenolic compounds, organic acids and sugars has been revealed and presented in the statistical distribution between these biochemicals.

\section{Materials and Methods}

\section{Fruits samples}

In this study, 'Aslik', 'Arapkizi', 'Bey Elmasi' and 'Cebegirmez' historical apple cultivars were used. These cultivars were grown in the Van region. They were harvested at full maturity stage. The fruit samples picked homogenously were stored at $-80^{\circ} \mathrm{C}$ until their laboratory analyses were conducted.

\section{Analysis of phenolic compounds}

In the research, the gallic acid, ellagic, catechin, chlorogenic acid, caffeic acid, syringic acid, $p$-coumaric acid, ferulic acid, $o$-coumaric acid, phloridzin, protocatechuic, vanillic acid, routine and quercetin were determined. In the separation of phenolic acids with HPLC (Agillent 1100 series HPLC G 1322 A, Germany), the method developed by Rodriguez-Delgado et al. (2001) was modified and used. The samples collected were distilled with distilled water at the ratio of 1:1 and after they were centrifuged at 15000 $\mathrm{rpm}$ for $15 \mathrm{~min}$., the supernatant was filtered with $0.45 \mu \mathrm{m}$ millipore filters and then injected to HPLC. The chromatographic separation was conducted by using DAD detector (Agilent. USA) and $250 * 4.6 \mathrm{~mm}, 4 \mu \mathrm{m}$ ODS colon (HiChrom, USA) in Agilent 1100 (Agilent) HPLC system. Solvent A Methanol-acidic acid-water (10:2:88), Solvent B Methanol-acidic acid-water (90:2:8) were used as the mobile phase. The separation was conducted at 254 and 280 $\mathrm{nm}$ and the flow rate was determined as $1 \mathrm{~mL} / \mathrm{min}$. and the injection volume was determined as $20 \mu \mathrm{L}$.

\section{Analysis of organic acids}

In the research, the citric acid, tartaric acid, malic acid, succinic acid, oxalic acid and fumaric acid contents were determined among the organic acids. In the extraction of organic acids, the method developed by Bevilacqua and
Califano (1989) was modified and used. $5 \mathrm{~g}$ was taken from the fruit samples obtained and transferred to centrifuge tubes. These samples were homogenized by adding $20 \mathrm{ml}$ 0.009 N H2SO 4 (Heidolph Silent Crusher M, Germany). Then, it was mixed on the agitator (Heidolph Unimax 1010, Germany) for 1 hour and centrifuged at $15000 \mathrm{rpm}$ for 15 minutes. The aqueous part which was separated at centrifuge was filtered from first coarse filter paper, then $0.45 \mu \mathrm{m}$ membrane filter (Millipore Millex-HV Hydrophilic PVDF, Millipore, USA) for two times and finally SEP-PAK C18 cartridge. The organic acids were analyzed in HPLC device (Agilent HPLC 1100 series G 1322 A, Germany) by using the method developed by Bevilacqua and Califano (1989). In HPLC system, Aminex HPX $-87 \mathrm{H}, 300 \mathrm{~mm} \times 7.8 \mathrm{~mm}$ colon (Bio-Rad Laboratories, Richmond, CA, USA) was used and the device was controlled with the computers including Agilent package program. DAD detector in the system (Agilent, USA) was set to 214 and $280 \mathrm{~nm}$ wavelengths. In the study, $0.009 \mathrm{~N} \mathrm{H}_{2} \mathrm{SO}_{4}$ filtered at $0.45 \mu \mathrm{m}$ membrane filter was used as mobile phase.

\section{Sugar analysis}

The modified method of Melgarejo et al. (2000) was used for sugar (fructose, glucose and sucrose) analyses. $5 \mathrm{~mL}$ of fruit extracts was centrifuged at $12000 \mathrm{rpm}$ for 2 minutes at temperature of $4{ }^{\circ} \mathrm{C}$. Supernatants were passed by SEPPAK C18 cartridge. HPLC readings were made with $\mu$ bondapak-NH2 column using $85 \%$ acetonitrile as liquid phase with refractive index detector (IR). Fructose and glucose standards were used for sugar calculations.

\section{Statistical analysis}

R 3.3.4 and SAS/STAT software was utilized for statistical analyzes of the data. Descriptive statistics, normal distribution tests, correlation analysis and one way variance analysis were performed in SAS software. One-way analysis of variance was used to determine the differences between local cultivars averages in terms of phenolic compounds, organic acids and sugars. The Duncan test was used as a multiple comparison test to express the differences between the averages. In $\mathrm{R}$ software, Principle component analysis (PCA) was used to for all variables examined using ggplot2 and facto extra packages (Package ggplot2, 2016; Package facto extra, 2017; SAS/STAT, 2012).

\section{Results and Discussion}

In this study, it was determined that the main phenolics were ellagic acid, followed by vanillic acid, routine and caffeic acid in historical apple cultivars (Tables 1, 2, 3, Fig. 1).

The highest ellagic acid content $(63.034 \mathrm{mg} / 100 \mathrm{ml})$ was obtained from 'Arapkizi' cultivar and followed by 'Cebegirmez' and 'Aslik' (Table 1). While the highest content of gallic and protocatechuic acid were recorded from 'Arapkizi', the lowest content was determined from 'Bey Elmasi' cultivar. 'Aslik' possessed the highest syringic $(2.792 \mathrm{mg} / 100 \mathrm{ml})$ and vanilic $(31.426 \mathrm{mg} / 100 \mathrm{ml})$ acid content (Table 1). Chen et al. (2012) determined that the percentage of gallic acid in the whole apple fruit is higher than the peel. He reported gallic acid content of apple 
624

cultivars in followed order: Red Delicious > Royal Gala > Golden Delicious. In a study of red and white fleshed apple cultivars, researchers reported that gallic and syringic content changed among cultivars. Gallic acid was measured as $4.0 \mathrm{mg} / \mathrm{kg}$ in red fleshed Hongxun and as $1.5 \mathrm{mg} / \mathrm{kg}$ in white flesh Golden Delicious. Also, syringic acid content ranged from 13.8 to $48.1 \mathrm{mg} / \mathrm{kg}$ (Wang et al., 2015). Although gallic acid values were comparable with our study, syringic acid values weren't comparable. Karadeniz et al. (2015) reported phenolic compound differences in apple cultivars. The difference with other studies is thought to be due to the cultivars, ecological factors and method differences.

The highest routine content was measured in 'Cebegirmez' as $21.020 \mathrm{mg} / 100 \mathrm{~g}$, but this cultivar possessed the lowest catechin and phloridzin content (Tables 1, 2, Fig. 1). Catechin content ranged from $4.347-12.619 \mathrm{mg} / 100 \mathrm{~g}$ among apple cultivars (Table 2). It was determined that the highest of phloridzin content was $4.513 \mathrm{mg} / 100 \mathrm{~g}$ as 'Arapkizi' (Table 2, Fig. 1). Jakobek et al. (2016) conducted a study on red and yellow peel colored apple cultivars and they measured phloridzin as $10.5-30.2 \mathrm{mg} / \mathrm{kg}$ in red peeled apple cultivars. Also, researchers found that yellow peeled apple cultivars had 3.0-13.7 mg/kg catechin content. However, contrary our study, some researchers reported that 'Arapkizi' had the lowest phloridzin content as 0.52 $\mathrm{mg} / \mathrm{kg}$ (Karaman et al., 2010). In another study, some researchers were recorded routine content as 0.37-1.59 $\mathrm{mg} / \mathrm{g}$ in whole fruit and $2.76-11.4 \mathrm{mg} / \mathrm{g}$ in apple peel in different cultivars (Lata et al., 2009). Schieber et al. (2015) conducted a study on phenolic component of apple juice and pomace. They determined that quercetin content measured as $1.2 \mathrm{mg} / \mathrm{L}$ in juice and $67 \mathrm{mg} / \mathrm{kg}$ in pomace. In another study, researchers reported that the highest catechin content recorded as $27.7 \mu \mathrm{g} / \mathrm{g}$ in SuperMac cultivar (Khanizadeh et al., 2008). It was observed that our values were higher than the results of some researches. It is thought that the difference is caused by ecological factors and cultivars.

The lowest phenolic compound among apple cultivars was measured as 0 -coumaric acid (Table 3, Fig. 1). 'Bey Elmasi' cultivar possessed the highest content of six phenolic

Table 1. Protocatechuic, vanillic, syringic, gallic and ellagic contents of historical apple cultivars (mg/100 ml)

\begin{tabular}{|c|c|c|c|c|c|}
\hline Variable & Historical cultivars & Mean & Low. 95\% & Upp. 95\% & $\begin{array}{c}\text { ANOVA } p \text {-value and R- } \\
\text { Square }\end{array}$ \\
\hline \multirow{4}{*}{ Ellagic } & Arapkizi & $63.084 \pm 2.961^{\mathrm{A}}$ & 25.461 & 100.707 & \multirow{4}{*}{$\begin{array}{c}<0.0001 \\
\mathrm{R}^{2}=0.9921\end{array}$} \\
\hline & Aslik & $23.859 \pm 0.503^{\mathrm{BC}}$ & 17.474 & 30.243 & \\
\hline & Bey Elmasi & $18.858 \pm 0.746^{\mathrm{C}}$ & 9.379 & 28.337 & \\
\hline & Cebegirmez & $26.835 \pm 0.357^{\mathrm{B}}$ & 22.299 & 31.371 & \\
\hline \multirow{4}{*}{ Gallic } & Arapkizi & $0.556 \pm 0.014^{\mathrm{A}}$ & 0.384 & 0.727 & \multirow{4}{*}{$\begin{array}{c}<0.0035 \\
\mathrm{R}^{2}=0.9564\end{array}$} \\
\hline & Aslik & $0.327 \pm 0.007^{\mathbf{B}}$ & 0.238 & 0.416 & \\
\hline & Bey Elmasi & $0.091 \pm 0.007^{\mathrm{C}}$ & 0.008 & 0.173 & \\
\hline & Cebegirmez & $0.228 \pm 0.071^{\mathrm{BC}}$ & -0.668 & 1.123 & \\
\hline \multirow{4}{*}{ Syringic } & Arapkizi & $0.896 \pm 0.007^{\mathrm{D}}$ & 0.813 & 0.978 & \multirow{4}{*}{$\begin{array}{c}<0.0001 \\
\mathrm{R}^{2}=0.9964\end{array}$} \\
\hline & Aslik & $2.792 \pm 0.007^{\mathrm{A}}$ & 2.703 & 2.881 & \\
\hline & Bey Elmasi & $2.187 \pm 0.079^{\mathbf{B}}$ & 1.189 & 3.184 & \\
\hline & Cebegirmez & $1.832 \pm 0.023^{\mathrm{C}}$ & 1.540 & 2.124 & \\
\hline \multirow{4}{*}{ Vanillic } & Arapkizi & $1.110 \pm 0.015^{\mathrm{C}}$ & 0.919 & 1.301 & \multirow{4}{*}{$\begin{array}{c}<0.0001 \\
\mathrm{R}^{2}=0.9991\end{array}$} \\
\hline & Aslik & $31.426 \pm 0.608^{A}$ & 23.701 & 39.151 & \\
\hline & Bey Elmasi & $23.751 \pm 0.443^{\mathrm{B}}$ & 18.128 & 29.373 & \\
\hline & Cebegirmez & $1.953 \pm 0.076^{\mathrm{C}}$ & 0.993 & 2.912 & \\
\hline \multirow{4}{*}{ Protocatechuic } & Arapkizi & $0.298 \pm 0.003^{\mathrm{A}}$ & 0.260 & 0.336 & \multirow{4}{*}{$\begin{array}{c}<0.001 \\
\mathrm{R}^{2}=0.9951\end{array}$} \\
\hline & Aslik & $0.259 \pm 0.001^{\mathrm{B}}$ & 0.252 & 0.265 & \\
\hline & Bey Elmasi & $0.153 \pm 0.006^{\mathrm{C}}$ & 0.077 & 0.229 & \\
\hline & Cebegirmez & $0.263 \pm 0.004^{\mathrm{B}}$ & 0.218 & 0.307 & \\
\hline
\end{tabular}

Table 2. Catechin, phloridzin, quercetin and routine contents of historical apple cultivars ( $\mathrm{mg} 100 / \mathrm{ml})$

\begin{tabular}{|c|c|c|c|c|c|}
\hline Variable & Historical cultivars & Mean & Low. 95\% & Upp. 95\% & $\begin{array}{l}\text { ANOVA } p \text {-value } \\
\text { and R-Square }\end{array}$ \\
\hline \multirow{4}{*}{ Catechin } & Arapkizi & $5.658 \pm 0.229^{C}$ & 2.748 & 8.568 & \multirow{4}{*}{$\begin{array}{c}<0.0001 \\
\mathrm{R}^{2}=0.9971\end{array}$} \\
\hline & Aslik & $6.751 \pm 0.194^{\mathrm{B}}$ & 4.292 & 9.209 & \\
\hline & Bey Elmasi & $12.619 \pm 0.138^{\mathrm{A}}$ & 10.871 & 14.366 & \\
\hline & Cebegirmez & $4.347 \pm 0.070^{\mathrm{D}}$ & 3.458 & 5.236 & \\
\hline \multirow{4}{*}{ Phloridzin } & Arapkizi & $4.513 \pm 0.015^{\mathrm{A}}$ & 4.322 & 4.704 & \multirow{4}{*}{$\begin{array}{c}<0.0022 \\
\mathrm{R}^{2}=0.9653\end{array}$} \\
\hline & Aslik & $3.332 \pm 0.166^{\mathrm{B}}$ & 1.229 & 5.434 & \\
\hline & Bey Elmasi & $3.441 \pm 0.075^{\mathrm{B}}$ & 2.488 & 4.394 & \\
\hline & Cebegirmez & $3.039 \pm 0.107^{\mathrm{B}}$ & 1.679 & 4.399 & \\
\hline \multirow{4}{*}{ Quercetin } & Arapkizi & $0.605 \pm 0.025^{\mathrm{B}}$ & 0.287 & 0.923 & \multirow{4}{*}{$\begin{array}{c}<0.0211 \\
\mathrm{R}^{2}=0.8918\end{array}$} \\
\hline & Aslik & $0.743 \pm 0.031^{\mathrm{A}}$ & 0.349 & 1.137 & \\
\hline & Bey Elmasi & $0.743 \pm 0.011^{\mathrm{A}}$ & 0.609 & 0.876 & \\
\hline & Cebegirmez & $0.650 \pm 0.007^{\mathrm{B}}$ & 0.567 & 0.732 & \\
\hline \multirow{4}{*}{ Routine } & Arapkizi & $13.451 \pm 1.211^{\mathrm{B}}$ & -1.936 & 28.838 & \multirow{4}{*}{$\begin{array}{c}<0.0006 \\
\mathrm{R}^{2}=0.9816\end{array}$} \\
\hline & Aslik & $8.336 \pm 0.805^{\mathrm{C}}$ & -1.892 & 18.564 & \\
\hline & Bey Elmasi & $4.150 \pm 0.742^{\mathrm{D}}$ & -5.278 & 13.578 & \\
\hline & Cebegirmez & $21.020 \pm 0.541^{\mathrm{B}}$ & 14.146 & 27.894 & \\
\hline
\end{tabular}


Table 3.p-Coumaric, ferulic, $o$-coumaric, chlorogenic and caffeic contents of apple cultivars $(\mathrm{mg} \mathrm{100/ml})$

\begin{tabular}{|c|c|c|c|c|c|}
\hline Variable & Historical cultivars & Mean & Low. 95\% & Upp. 95\% & $\begin{array}{c}\text { ANOVA p-value and } \\
\text { R-Square }\end{array}$ \\
\hline \multirow{4}{*}{ Caffeic } & Arapkizi & $7.298 \pm 0.081^{\mathbf{B}}$ & 6.275 & 8.320 & \multirow{4}{*}{$\begin{array}{c}<0.0001 \\
\mathrm{R}^{2}=0.9980\end{array}$} \\
\hline & Aslik & $7.740 \pm 0.039^{\mathbf{B}}$ & 7.244 & 8.236 & \\
\hline & Bey Elmasi & $6.947 \pm 0.083^{\mathrm{B}}$ & 5.898 & 7.995 & \\
\hline & Cebegirmez & $22.743 \pm 0.583^{\mathrm{A}}$ & 15.341 & 30.144 & \\
\hline \multirow{4}{*}{ Chlorogenic } & Arapkizi & $3.594 \pm 0.053^{\mathrm{C}}$ & 2.921 & 4.267 & \multirow{4}{*}{$\begin{array}{c}<0.0001 \\
\mathrm{R}^{2}=0.9985\end{array}$} \\
\hline & Aslik & $3.919 \pm 0.020^{\mathrm{B}}$ & 3.665 & 4.173 & \\
\hline & Bey Elmasi & $5.630 \pm 0.049^{A}$ & 5.013 & 6.246 & \\
\hline & Cebegirmez & $2.348 \pm 0.050^{\mathbf{D}}$ & 1.713 & 2.983 & \\
\hline \multirow{4}{*}{ Ferulic } & Arapkizi & $0.355 \pm 0.008^{\mathrm{AB}}$ & 0.253 & 0.457 & \multirow{4}{*}{$\begin{array}{c}<0.0253 \\
\mathrm{R}^{2}=0.8814\end{array}$} \\
\hline & Aslik & $0.477 \pm 0.003^{\mathrm{A}}$ & 0.445 & 0.508 & \\
\hline & Bey Elmasi & $0.500 \pm 0.035^{\mathrm{A}}$ & 0.055 & 0.945 & \\
\hline & Cebegirmez & $0.243 \pm 0.067^{\mathrm{B}}$ & -0.602 & 1.087 & \\
\hline \multirow{4}{*}{ p_coumaric } & Arapkizi & $1.105 \pm 0.085^{\mathrm{A}}$ & 0.025 & 2.185 & \multirow{4}{*}{$\begin{array}{c}<0.0001 \\
\mathrm{R}^{2}=0.9958\end{array}$} \\
\hline & Aslik & $0.966 \pm 0.006^{\mathrm{A}}$ & 0.896 & 1.035 & \\
\hline & Bey Elmasi & $1.112 \pm 0.003^{\mathrm{A}}$ & 1.080 & 1.143 & \\
\hline & Cebegirmez & $0.952 \pm 0.005^{\mathrm{A}}$ & 0.894 & 1.009 & \\
\hline \multirow{4}{*}{ o_coumaric } & Arapkizi & $0.091 \pm 0.008^{\mathrm{C}}$ & -0.005 & 0.186 & \multirow{4}{*}{$\begin{array}{c}<0.1022 \\
\mathrm{R}^{2}=0.7558\end{array}$} \\
\hline & Aslik & $0.082 \pm 0.004^{\mathrm{C}}$ & 0.037 & 0.126 & \\
\hline & Bey Elmasi & $0.267 \pm 0.005^{\mathrm{A}}$ & 0.209 & 0.324 & \\
\hline & Cebegirmez & $0.163 \pm 0.002^{\mathrm{B}}$ & 0.143 & 0.182 & \\
\hline
\end{tabular}

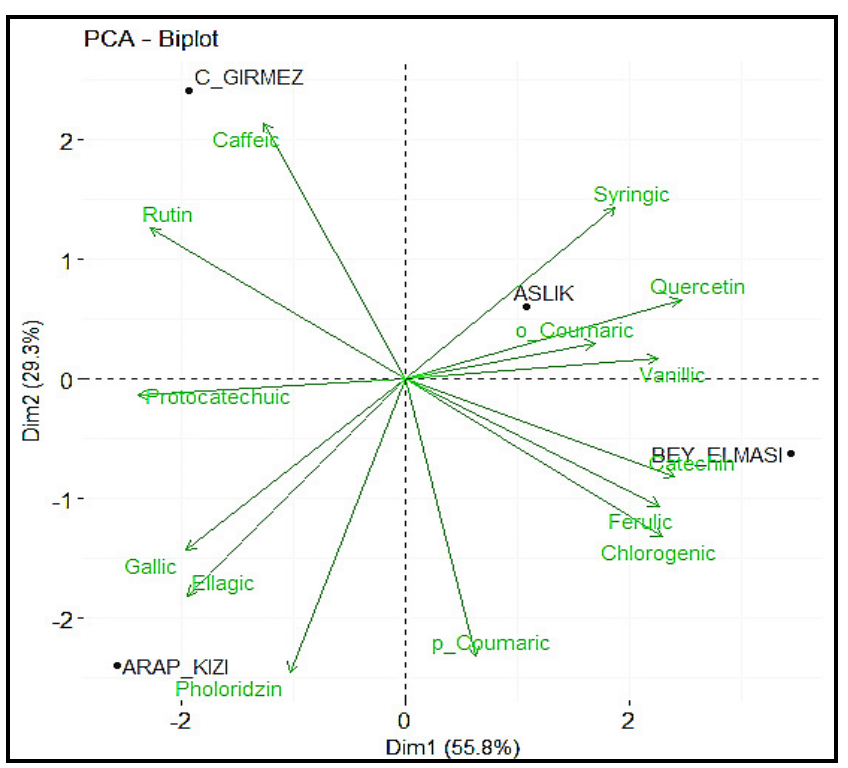

Fig. 1. The distribution of phenolic compounds according to cultivars

acids but this cultivar also had the lowest content of five the other phenolic compounds (Tables $1,2,3$ ). In addition 'Arapkizi' cultivar had the highest content of five phenolic acids (Tables 1, 2,3).

It was identified that there was a positive correlation among gallic, ellagic acid and routine (Table 4, Fig. 2). However there was a negative correlation between gallic and ellagic acid and syringic acid. Therefore, ellagic acid increased as gallic acid increased but syringic acid decreased (Table 4, Fig.2). A similar positive correlation was also identified among ferulic, catechin and chlorogenic acids (Table 4, Fig. 2). But these acids had a negative correlation with routine (Table 4, Fig.2). As shown in Table 1, there was a statistical difference among historical apple cultivars for phenolic acids.

The highest o-coumaric, chlorogenic, ferulic and $p$ coumaric acid content were obtained from 'Bey Elmasi' cultivar (Table 3), the lowest content was obtained from 'Cebegirmez'. Caffeic acid content ranged from 6.947$22.743 \mathrm{mg} / 100 \mathrm{ml}$ and 'Cebegirmez' cultivar possessed this highest content (Table 3). The highest chlorogenic content was measured as $5.630 \mathrm{mg} / 100 \mathrm{ml}$ (Table 3). Coşkun et al. (2016) reported chlorogenic acid ranged between 29.15 $\mathrm{mg} / \mathrm{kg}$ and $194.45 \mathrm{mg} / \mathrm{kg}$ in some local apple cultivars. Also researchers determined the highest caffeic acid was 8.18 $\mathrm{mg} / \mathrm{kg}$. In Turkey, chlorogenic and caffeic acid content of 'Arapkizi' were measured as 242.3 and $12.14 \mathrm{mg} / \mathrm{L}$, respectively by Karaman et al. (2010). In both studies, caffeic acid values are in agreement with our study but chlorogenic acid values are not comparable. In a study by using apple cultivars, the amount of ferulic acid content was found to be higher than $p$-coumaric acid and the ratio of these acids changed for all cultivars (Chen et al. 2012). Wang et al. (2015) determined that the highest $p$-coumaric, ferulic, caffeic and chlorogenic acid content measured as 0.5 , $0.5,1.3$ and $196.0 \mathrm{mg} / \mathrm{kg}$. Khanizadeh et al. (2007) measured chlorogenic acid as $183.1 \mu \mathrm{g} / \mathrm{g}$ and phloridzin as $11.8 \mu \mathrm{g} / \mathrm{g}$ in Gala while Tsao et al. (2003) measured chlorogenic acid as $153.6 \mu \mathrm{g} / \mathrm{g}$ and phloridzin as $17.96 \mu \mathrm{g} / \mathrm{g}$ in Golden Delicious.

As shown Table 5 and Fig. 3, there was a difference between cultivars in terms of sugar values. It was determined that fructose was the major sugar for all apple cultivars. It was followed by glucose and sucrose. In all sugars, 'Aslik' cultivar had the highest sugar content while 'Arapkizi' had the lowest sugar content (Table 5, Fig. 3). The content of fructose ranged from $12.315 \mathrm{~g} / \mathrm{L}$ ('Arapkizi') to $16.495 \mathrm{~g} / \mathrm{L}$ ('Aslik') (Table 5, Fig. 3). It was obtained the amount of fructose and glucose was 10 times higher than sucrose 
626

Table 4. Correlations between phenolic compounds

\begin{tabular}{|c|c|c|c|c|c|c|c|c|c|c|c|c|c|c|}
\hline \multicolumn{15}{|c|}{ Pearson Correlation Coefficients, $\mathrm{N}=8$} \\
\hline & Protocatechuic & Vanillic & Ellagic & Routine & Quercetin & Gallic & Catechin & Chlorogenic & Caffeic & Syringic & p_Coumaric & Ferulic & $o_{-}$Coumaric & Pholoridzin \\
\hline \multirow{2}{*}{ Protocatechuic } & 1.000 & -0.532 & 0.697 & 0.653 & -0.666 & $0.838^{*}$ & $-0.921^{*}$ & $-0.794^{*}$ & 0.232 & -0.451 & -0.296 & -0.556 & $-0.902^{*}$ & 0.400 \\
\hline & P-Value & 0.175 & 0.055 & 0.079 & 0.071 & 0.009 & 0.001 & 0.019 & 0.581 & 0.262 & 0.477 & 0.153 & 0.002 & 0.326 \\
\hline \multirow{2}{*}{ Vanillic } & & 1.000 & -0.653 & $-0.790^{*}$ & $0.893^{*}$ & -0.432 & 0.587 & 0.639 & -0.535 & $0.875^{*}$ & -0.078 & $0.819^{*}$ & 0.141 & -0.366 \\
\hline & P-Value & & 0.079 & 0.020 & 0.003 & 0.285 & 0.126 & 0.088 & 0.172 & 0.005 & 0.855 & 0.013 & 0.739 & 0.372 \\
\hline \multirow{2}{*}{ Ellagic } & & & 1.000 & 0.308 & $-0.781^{*}$ & $0.889^{*}$ & -0.452 & -0.296 & -0.205 & $-0.870^{*}$ & 0.401 & -0.320 & -0.550 & $0.896^{*}$ \\
\hline & P-Value & & & 0.458 & 0.022 & 0.003 & 0.261 & 0.476 & 0.626 & 0.005 & 0.325 & 0.440 & 0.158 & 0.003 \\
\hline \multirow{2}{*}{ Routine } & & & & 1.000 & -0.687 & 0.294 & $-0.850^{*}$ & $-0.946^{*}$ & $0.853^{*}$ & -0.437 & -0.405 & $-0.918^{*}$ & -0.336 & -0.095 \\
\hline & P-Value & & & & 0.060 & 0.480 & 0.008 & 0.000 & 0.007 & 0.279 & 0.320 & 0.001 & 0.416 & 0.823 \\
\hline \multirow{2}{*}{ Quercetin } & & & & & 1.000 & -0.669 & 0.636 & 0.600 & -0.321 & $0.871^{*}$ & -0.017 & $0.708^{*}$ & 0.392 & -0.573 \\
\hline & P-Value & & & & & 0.070 & 0.090 & 0.116 & 0.438 & 0.005 & 0.968 & 0.049 & 0.337 & 0.137 \\
\hline \multirow{2}{*}{ Gallic } & & & & & & 1.000 & -0.606 & -0.397 & -0.215 & -0.607 & 0.088 & -0.309 & $-0.823^{*}$ & $0.782^{*}$ \\
\hline & P-Value & & & & & & 0.111 & 0.330 & 0.610 & 0.111 & 0.836 & 0.457 & 0.012 & 0.022 \\
\hline \multirow{2}{*}{ Catechin } & & & & & & & 1.000 & $0.962^{*}$ & -0.570 & 0.331 & 0.546 & $0.735^{*}$ & $0.765^{*}$ & -0.067 \\
\hline & P-Value & & & & & & & 0.000 & 0.140 & 0.423 & 0.161 & 0.038 & 0.027 & 0.874 \\
\hline \multirow{2}{*}{ Chlorogenic } & & & & & & & & 1.000 & $-0.769^{*}$ & 0.288 & 0.580 & $0.828^{*}$ & 0.571 & 0.120 \\
\hline & P-Value & & & & & & & & 0.026 & 0.489 & 0.132 & 0.011 & 0.139 & 0.776 \\
\hline \multirow{2}{*}{ Caffeic } & & & & & & & & & 1.000 & -0.064 & -0.568 & $-0.808^{*}$ & 0.060 & -0.550 \\
\hline & P-Value & & & & & & & & & 0.881 & 0.142 & 0.015 & 0.887 & 0.157 \\
\hline \multirow{2}{*}{ Syringic } & & & & & & & & & & 1.000 & -0.443 & 0.504 & 0.154 & $-0.742^{*}$ \\
\hline & P-Value & & & & & & & & & & 0.271 & 0.203 & 0.716 & 0.035 \\
\hline \multirow{2}{*}{ P_coumaric } & & & & & & & & & & & 1.000 & 0.336 & 0.354 & 0.610 \\
\hline & P-Value & & & & & & & & & & & 0.416 & 0.390 & 0.108 \\
\hline \multirow{2}{*}{ Ferulic } & & & & & & & & & & & & 1.000 & 0.213 & 0.001 \\
\hline & P-Value & & & & & & & & & & & & 0.613 & $0.998^{*}$ \\
\hline \multirow{2}{*}{${ }_{\text {o_coumaric }}$} & & & & & & & & & & & & & 1.000 & -0.364 \\
\hline & P-Value & & & & & & & & & & & & & 0.375 \\
\hline Pholoridzin & & & & & & & & & & & & & & 1.000 \\
\hline
\end{tabular}

${ }^{*}: \mathrm{P} \leq 0.001$

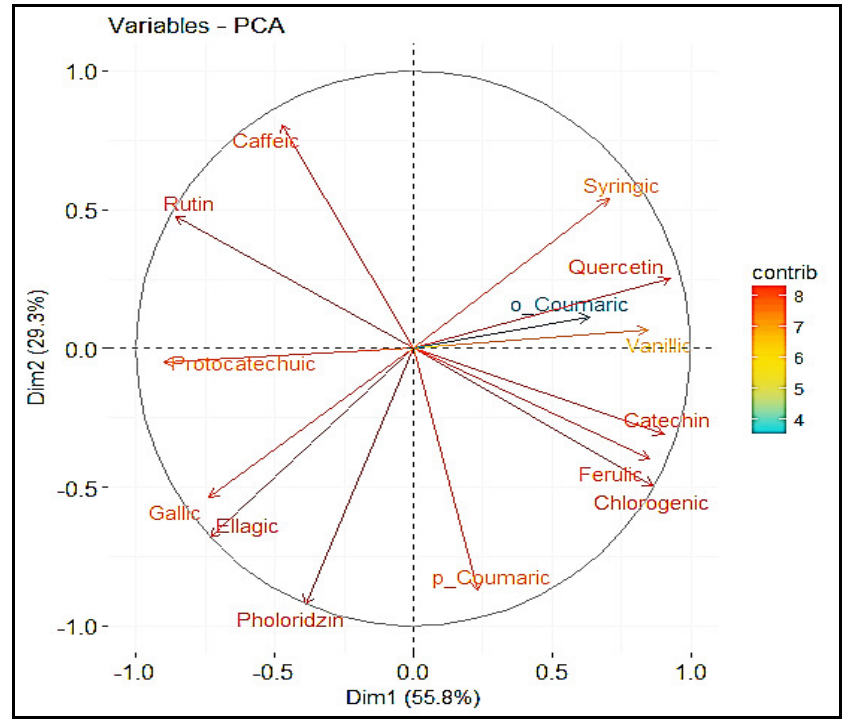

Fig. 2. The correlation of phenolic compounds

content. Referring to Table 7 and Fig. 4, there was a positive correlation among fructose, glucose and sucrose content. So, it was seen that glucose and sucrose content increased as fructose content increased (Table 7, Fig. 4). In Portugal, researchers searched phytochemicals of some apple cultivars. In parallel with our study, it was determined that the major sugar was fructose (4.23-5.62 g/L). Also, researchers measured glucose and sucrose content as $0.73-1.95,1.17$ $2.98 \mathrm{~g} / \mathrm{L}$ respectively (Feliciano $e t$ al., 2010). In a different study, sugar concentrations varied depending on the cultivars. Fructose and sucrose concentrations were measured as major sugar. Researchers reported that glucose ranged $5-20 \mathrm{~g} / \mathrm{kg}$ (Hecke et al., 2006). Our findings are higher compared to Hecke et al. (2006) work.
When the organic acid values were examined, statistically differences between the cultivars were significant $(p<0.05)$ (Table 6). Malic acid was measured as the major organic acid while tartaric acid was measured as the lowest (Table 6, Fig. 3). The organic acid contents were as follows: malic $>$ fumaric $>$ succinic $>$ citric $>$ tartaric (Table 6, Fig. 3). 'Arapkizi' cultivar possessed the highest value of four organic acids except of tartaric acid (Table 6). On the contrary, 'Bey Elmasi' cultivar had the lowest content of organic acids (Table 6). The highest malic acid and fumaric acid content was recorded as $4.613 \mathrm{mg} / 100 \mathrm{ml}$ and 1.252 $\mathrm{mg} / 100 \mathrm{ml}$ ('Arapkizi') (Table 6), respectively. It was determined that 'Cebegirmez' cultivar had the lowest succinic $(0.144 \mathrm{mg} / 100 \mathrm{ml})$ and malic acid content but the highest tartaric acid as $0.214 \mathrm{mg} / 100 \mathrm{ml}$ (Table 6). As shown Table 7 and Fig. 4, the positive correlation was observed among malic, fumaric and succinic acid. No relationship was found between these acids and tartaric and malic acid. However the opposite correlation between citric acid and all sugars was obtained. That is, fructose, glucose and sucrose decreased as citric acid increased (Table 7, Fig. 4). According to researchers, the highest organic acid content was measured as $7106.05 \mathrm{mg} / \mathrm{kg}$ (as malic acid) of some local apple cultivars. They determined that tartaric acid ranged between $84.00-382.55 \mathrm{mg} / \mathrm{kg}$ (Coşkun et al., 2016). Feliciano et al. (2010) reported that citric acid content ranged from $2.8-8.7 \mathrm{mg} / 100 \mathrm{~g}$ and the major organic acid was malic acid in apple. In a study conducted in Austria, it was determined that malic acid was a major acid and ranged from $5.64-17.8 \mathrm{~g} / \mathrm{kg}$. Also, fumaric acid was identified at the lowest organic acid (Hecke et al., 2006). Malic acid was found major acid in all studies, in parallel with our research. However there are numerical differences with other studies. This difference is thought to have originated from the cultivars and ecology. 
Table 5. Fructose, glucose and sucrose content of apple cultivars $\left(\mathrm{g}^{-1}\right)$

\begin{tabular}{|c|c|c|c|c|c|}
\hline Variable & Historical cultivars & Mean & $\begin{array}{l}\text { Low. } \\
95 \%\end{array}$ & $\begin{array}{l}\text { Upp. } \\
95 \%\end{array}$ & $\begin{array}{c}\text { ANOVA p-value and R- } \\
\text { Square }\end{array}$ \\
\hline \multirow{4}{*}{ Fructose } & Arapkizi & $12.315 \pm 0.019^{\mathrm{D}}$ & 12.074 & 12.556 & \multirow[t]{4}{*}{$<0.0001 \mathrm{R}^{2}=0.9957$} \\
\hline & Aslik & $16.495 \pm 0.156^{\mathrm{A}}$ & 14.513 & 18.477 & \\
\hline & Bey Elmasi & $15.497 \pm 0.125^{\mathrm{B}}$ & 13.915 & 17.078 & \\
\hline & Cebegirmez & $13.818 \pm 0.054^{\mathrm{C}}$ & 13.132 & 14.504 & \\
\hline \multirow{4}{*}{ Glucose } & Arapkizi & $10.461 \pm 0.067^{D}$ & 9.616 & 11.305 & \multirow[t]{4}{*}{$<0.0002 \mathrm{R}^{2}=0.9908$} \\
\hline & Aslik & $12.739 \pm 0.045^{\mathrm{A}}$ & 12.173 & 13.304 & \\
\hline & Bey Elmasi & $12.073 \pm 0.142^{\mathrm{B}}$ & 10.275 & 13.870 & \\
\hline & Cebegirmez & $11.255 \pm 0.028^{\mathrm{C}}$ & 10.899 & 11.611 & \\
\hline \multirow{4}{*}{ Sucrose } & Arapkizi & $0.179 \pm 0.005^{\mathrm{D}}$ & 0.115 & 0.243 & \multirow{4}{*}{$<0.0001 \mathrm{R}^{2}=0.9918$} \\
\hline & Aslik & $0.423 \pm 0.012^{\mathrm{A}}$ & 0.271 & 0.575 & \\
\hline & Bey Elmasi & $0.337 \pm 0.010^{\mathbf{B}}$ & 0.210 & 0.464 & \\
\hline & Cebegirmez & $0.243 \pm 0.004^{\mathrm{C}}$ & 0.198 & 0.287 & \\
\hline
\end{tabular}

Table 6. Organic acid content of apple cultivars $(\mathrm{mg} / 100 \mathrm{ml})$

\begin{tabular}{|c|c|c|c|c|c|}
\hline Variable & Local cultivars & Mean & $\begin{array}{l}\text { Low. } \\
95 \%\end{array}$ & $\begin{array}{l}\text { Upp. } \\
95 \%\end{array}$ & $\begin{array}{c}\text { ANOVA p-value and R- } \\
\text { Square }\end{array}$ \\
\hline \multirow{4}{*}{ Citric } & Arapkizi & $0.447 \pm 0.002^{\mathrm{A}}$ & 0.422 & 0.472 & \multirow{4}{*}{$\begin{array}{c}<0.0001 \\
\mathrm{R}^{2}=0.9996\end{array}$} \\
\hline & Aslik & $0.109 \pm 0.002^{\mathrm{D}}$ & 0.089 & 0.128 & \\
\hline & Bey Elmasi & $0.376 \pm 0.005^{\mathrm{B}}$ & 0.331 & 0.420 & \\
\hline & Cebegirmez & $0.275 \pm 0.002^{\mathrm{C}}$ & 0.255 & 0.294 & \\
\hline \multirow{4}{*}{ Fumaric } & Arapkizi & $1.252 \pm 0.128^{\mathrm{A}}$ & -0.374 & 2.878 & \multirow{4}{*}{$\begin{array}{c}<0.0081 \\
\mathrm{R}^{2}=0.9337\end{array}$} \\
\hline & Aslik & $0.972 \pm 0.004^{\mathrm{B}}$ & 0.921 & 1.023 & \\
\hline & Bey Elmasi & $0.634 \pm 0.009^{\mathrm{C}}$ & 0.520 & 0.748 & \\
\hline & Cebegirmez & $0.718 \pm 0.007^{\mathrm{C}}$ & 0.629 & 0.807 & \\
\hline \multirow{4}{*}{ Malic } & Arapkizi & $4.613 \pm 0.008^{\mathrm{A}}$ & 4.511 & 4.715 & \multirow{4}{*}{$\begin{array}{c}<0.0001 \\
\mathrm{R}^{2}=0.9935\end{array}$} \\
\hline & Aslik & $3.915 \pm 0.045^{\mathrm{B}}$ & 3.343 & 4.487 & \\
\hline & Bey Elmasi & $3.195 \pm 0.045^{\mathrm{C}}$ & 2.623 & 3.767 & \\
\hline & Cebegirmez & $3.095 \pm 0.075^{\mathrm{C}}$ & 2.142 & 4.048 & \\
\hline \multirow{4}{*}{ Succinic } & Arapkizi & $0.511 \pm 0.004^{\mathrm{A}}$ & 0.466 & 0.555 & \multirow{4}{*}{$\begin{array}{c}<0.0001 \\
\mathrm{R}^{2}=0.9990\end{array}$} \\
\hline & Aslik & $0.419 \pm 0.002^{\mathrm{B}}$ & 0.394 & 0.444 & \\
\hline & Bey Elmasi & $0.361 \pm 0.007^{\mathrm{C}}$ & 0.278 & 0.443 & \\
\hline & Cebegirmez & $0.144 \pm 0.003^{\mathrm{D}}$ & 0.106 & 0.182 & \\
\hline \multirow{4}{*}{ Tartaric } & Arapkizi & $0.130 \pm 0.007^{\mathrm{B}}$ & 0.047 & 0.212 & \multirow{4}{*}{$\begin{array}{c}<0.0002 \\
\mathrm{R}^{2}=0.9889\end{array}$} \\
\hline & Aslik & $0.140 \pm 0.003^{\mathrm{B}}$ & 0.102 & 0.178 & \\
\hline & Bey Elmasi & $0.075 \pm 0.003^{\mathrm{C}}$ & 0.037 & 0.113 & \\
\hline & Cebegirmez & $0.214 \pm 0.007^{\mathrm{A}}$ & 0.125 & 0.303 & \\
\hline
\end{tabular}

Table 7. Correlations between organic acids and sugars

\begin{tabular}{|c|c|c|c|c|c|c|c|c|}
\hline \multicolumn{9}{|c|}{ Pearson Correlation Coefficients, $\mathrm{N}=8$} \\
\hline & Citric & Tartaric & Malic & Succinic & Fumaric & Fructose & Glucose & Sucrose \\
\hline \multirow{2}{*}{ Citric } & 1.000 & -0.328 & 0.224 & 0.239 & 0.179 & $-0.749^{*}$ & $-0.781^{*}$ & $-0.783^{*}$ \\
\hline & P-value & 0.428 & 0.593 & 0.568 & 0.671 & 0.032 & 0.022 & 0.022 \\
\hline \multirow{2}{*}{ Tartaric } & & 1.000 & -0.182 & -0.652 & 0.018 & -0.289 & -0.263 & -0.291 \\
\hline & P-value & & 0.666 & 0.080 & 0.967 & 0.488 & 0.530 & 0.485 \\
\hline \multirow{2}{*}{ Malic } & & & 1.000 & $0.844^{*}$ & $0.950^{*}$ & -0.422 & -0.388 & -0.300 \\
\hline & P-value & & & 0.008 & 0.000 & 0.298 & 0.342 & 0.470 \\
\hline \multirow{2}{*}{ Succinic } & & & & 1.000 & $0.718^{*}$ & -0.072 & -0.066 & 0.030 \\
\hline & P-value & & & & 0.045 & 0.865 & 0.877 & 0.943 \\
\hline \multirow{2}{*}{ Fumaric } & & & & & 1.000 & -0.482 & -0.453 & -0.365 \\
\hline & P-value & & & & & 0.227 & 0.260 & 0.375 \\
\hline \multirow{2}{*}{ Fructose } & & & & & & 1.000 & $0.990^{*}$ & $0.983^{*}$ \\
\hline & P-value & & & & & & $<.0001$ & $<.0001$ \\
\hline \multirow{2}{*}{ Glucose } & & & & & & & 1.000 & $0.981^{*}$ \\
\hline & P-value & & & & & & & $<.0001$ \\
\hline Sucrose & & & & & & & & 1.000 \\
\hline
\end{tabular}


628

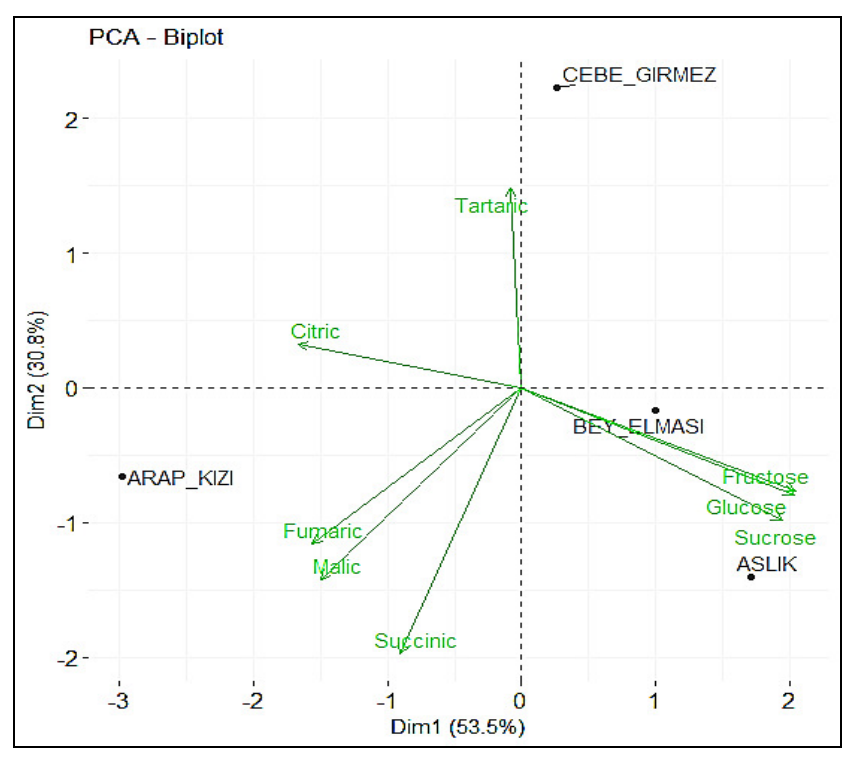

Fig. 3 The distribution of sugar and organic acid according to cultivars

\section{Conclusions}

As a result of the study, it was determined that 'Arapkizi' cultivar came into prominence the organic acid and phenolic contents. In the study, it was found that phenolic compounds explain $85.1 \%$ of the total variation with two basic components. In terms of the cultivars, pholoridzin, ellagic and cholorgenic acids were found to be the most potent compounds while vanillic and $o$-coumaric acid were found to be the lowest potent compounds. Catechin, ferulic and chlorogenic acids have been found to be highly correlated compounds among themselves, and negative correlation with caffeic and routine. In this study, $83.3 \%$ of the variation in organic acid and sugar content of the cultivars was explained by two main components. Positive correlations exist between organic acids and the highest positive correlations were observed between succinic, malik and fumaric acids. 'Aslik', 'Bey Elmasi' and 'Cebegirmez' local cultivars have the highest content of sugar while the 'Arapkizi' cultivar has been identified rich in organic acids. It was concluded that these two cultivars ('Aslik' and 'Arapkizi') would be useful for human health. As a result, the production of these cultivars should be increased.

\section{Acknowledgements}

This study was founded by Head of Scientific Research of Yuzuncu Y1l University, Project No: 2012 ZF B016.

\section{References}

Bevilacqua AE, Califano N (1989). Determination of organic acids in dairy products by high performance liquid chromatography. Journal of Food Science 54:1076-1079.

Boyer J, Liu RH (2004). Apple phytochemicals and their health benefits. Nutrition Journal 3:2-15.

Caliskan O, Bayazit S, Oktem M, Ergul A (2017). Evaluation of the genetic

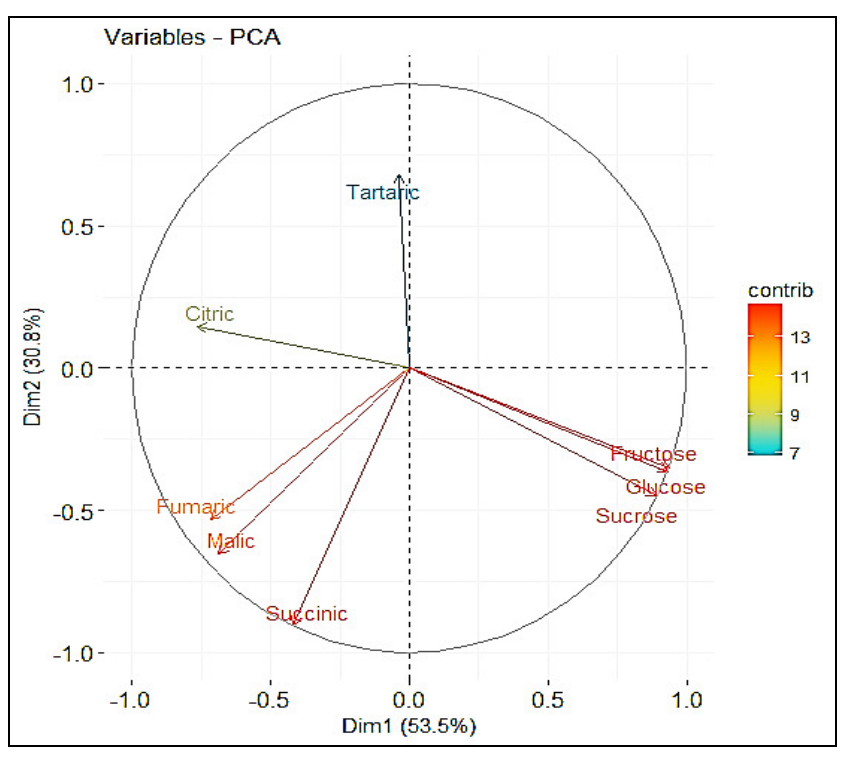

Fig. 4. The correlation of sugar and organic acids

diversity of pomegranate accessions from Turkey using new microsatellite markers. Turkish Journal of Agriculture and Forestry 41:142-153.

Canan I, Gundogdu M, Seday U, Oluk CA, Karasahin Z, Eroglu EC, YaziciE, Unlu M(2016). Determination of antioxidant, total phenolic, total carotenoid, lycopene, ascorbic acid, and sugar contents of Citrus species and mandarin hybrids. Turkish Journal of Agriculture and Forestry 40:894-899.

Carbone K, Giannini B, Picchi V, Lo Scalzo R, Cecchini F (2011). Phenolic composition and free radical scavenging activity of different apple varieties in relation to the cultivar, tissue type and storage. Food Chemistry 127:493-500.

Chen CS, Zhang D, Wang YQ, Li PM, Ma FW (2012). Effects of fruit bagging on the contents of phenolic compounds in the peel and flesh of 'Golden Delicious', 'Red Delicious', and 'Royal Gala' apples. Scientia Horticulturae 142:68-73.

Coşkun S, Aşkın MA (2016). Bazı yerli elma çeşitlerinin pomolojik ve biyokimyasal özelliklerinin belirlenmesi. Journal of Suleyman Demirel University Agricultural Faculty 11:120-131.

ErcisliS, Tosun M, Karlidag H, Dzubur A, HadziabulicS, Aliman Y(2012). Color and antioxidant characteristics of some fresh fig (Ficus carica L.) genotypes from Northeastern Turkey. Plant Foods for Human Nutrition 67:271-276.

FAO (2017). Food and Agricultural Organization of the United Nations. http://www.fao.org/faostat/en/\#data/QC.

Feliciano RP, Antunes C, Ramos A, Serra AT, Figueira ME, Duarte CMM, Carvalho A, Bronze MR (2010). Characterization of traditional and exotic apple varieties from Portugal. Part 1 - Nutritional, phytochemical and sensory evaluation. Journal of Functional Foods 2:35-45.

HeckeK, Herbinger K, Veberic R, Trobec M, Toplak H, Stampar F, Keppel H, Grill D (2006). Sugar-, acid- and phenol contents in apple cultivars from organic and integrated fruit cultivation. European Journal of Clinical Nutrition 60:1136-1140.

Jakobek L, Barron AR (2016). Ancient apple varieties from Croatia as a 
source of bioactive polyphenolic compounds. Journal of Food Composition and Analysis 45:9-15.

Kamiloglu O, Ercisli S, Sengul M, Toplu C, Serce S (2009). Total phenolics and antioxidant activity of jujube (Zizyphus jujube Mill.) genotypes selected from Turkey. African Journal of Biotechnology 8:303-307.

Karadeniz F. Burdurlu HS, Koca N, Soyer Y (2005). Antioxidant activity of selected fruits and vegetables grown in Turkey. Turkish Journal of Agriculture andForestry 29:297-303.

Karaman Ş, Tütem E, Sözgen Başkan K, Apak R (2010). Comparison of total antioxidant capacity and phenolic composition of some apple juices with combined HPLC-CUPRAC assay. Food Chemistry 120:12011209.

Kaya T, Balta F (2013). Apple selection in Van province. Agriculture Academy Journal 2:91-98.

Khanizadeh S, Tsao R, Rekika D, Yang R, DeEll J (2007). Phenolic composition and antioxidant activity of selected apple genotypes. Journal of Food Agriculture and Environment 5:61-66.

Khanizadeh S, Tsao R, Rekika D, Yang R, Charles MT, Rupasinghe HPV (2008). Polyphenol composition and total antioxidant capacity of selected apple genotypes for processing. Journal of Food Composition and Analysis 21:396-401.

Lata B, Trampczynska A, Paczesna J (2009). Cultivar variation in apple peel and whole fruit phenolic composition. Scientia Horticulturae 121:176181.

Melgarejo P, Salazar DM, Artes F (2000). Organic acids and sugars composition of harvested pomegranate fruits. European Food Research and Technology211:185-190.

Michels KB, Giovannucci E, Chan AT, Singhania R, Fuchs CS, Willett WC (2006). Fruit and vegetable consumption and colorectal adenomas in nurses' health study. Cancer Research 66:3942-3953.

Mordogan N, Ergun S (2001). Relationships between organic acids and minerals in apple fruits. Ege University Agricultural Faculty Journal 38:111-118.

Özrenk K, Gündogdu M, Kaya T, Kan T (2011). Pomological classification of apples grown in Çatak and Tatvan. Yuzuncu Yll University Agricultural Science Journal 21:57-63.
Package factoextra (2017). https://cran.r-project.org/web /packages /factoextra/factoextrapdf.

Package ggplot 2 (2016). https://cran.r-project.org/web/packa ges /ggplot2/ggplot2.pdf.

Rodriguez-Delgado MA, Malovana S, Perez JP, Borges T, GarciaMontelongo FJ (2001). Separation of phenolic compounds by highperformance liquid chromatography with absorbance and fluorimetric detection. Journal of Chromatography A 912:249-257.

SAS/STAT (2012). SAS Institute Inc. Cary, NC, USA.

Schieber A, Keller P, Carle R (2001). Determination of phenolic acids and flavonoids of apple and pear by high-performance liquid chromatography.Journal of Chromatography A 910:265-273.

Seçmen T, Onursal CE, Küçükyumuk C, Eren I, Güneyli A, Çalhan Ö (2015). The effect of deficit irrigation on storage performance and phenolic compounds in Breaburn apples. Fruit Science 2:16-21.

Sesso H, Gaziano JM, Liu S, Buring J (2003). Flavonoid intake and risk of cardiovascular disease in women. American Journal of Clinical Nutrition 77:1400-1408.

Tsao R, Yang R, Young JC, Zhu H (2003). Polyphenolic profiles in eight apple cultivars using High-Performance Liquid Chromatography (HPLC).Journal of Agricultural and Food 51:6347-6353.

Tosun M, Ercisli S, Karlidag H, Sengul M (2009). Characterization of red raspberry (Rubus idaeus L.) genotypes for their physicochemical properties. Journal of Food Science 74:C575-C579.

Wang X, Li C, Liang D, Zou Y, Li P, Ma F (2015). Phenolic compounds and antioxidant activity in red-fleshed apples. Journal of Functional Foods 18:1086-1094.

Wolfe K, Wu X, Liu RH (2003). Antioxidant activity of apple peels. Journal of Agricultural and Food Chemistry 51:609-614.

Xu Y, Fan M, Ran J, Zhang T, Sun H, DongM, ZhangZ,ZhengH(2016). Variation in phenolic compounds and antioxidant activity in apple seeds of seven cultivars. Saudi Journal of Biological Sciences 23:379-388. 